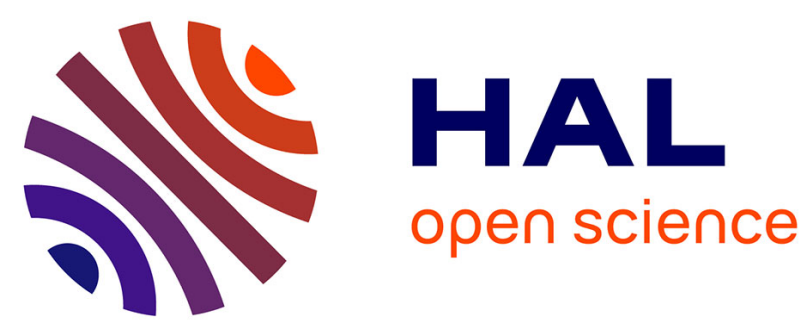

\title{
Kinematics, Workspace and Singularity Analysis of a Parallel Robot with Five Operation Modes *
}

Damien Chablat, Xianwen Kong, Chengwei Zhang

\section{To cite this version:}

Damien Chablat, Xianwen Kong, Chengwei Zhang. Kinematics, Workspace and Singularity Analysis of a Parallel Robot with Five Operation Modes *. Journal of Mechanisms and Robotics, 2018, 10 (3), 10.1115/1.4039400 . hal-01797872

HAL Id: hal-01797872

https://hal.science/hal-01797872

Submitted on 22 May 2018

HAL is a multi-disciplinary open access archive for the deposit and dissemination of scientific research documents, whether they are published or not. The documents may come from teaching and research institutions in France or abroad, or from public or private research centers.
L'archive ouverte pluridisciplinaire HAL, est destinée au dépôt et à la diffusion de documents scientifiques de niveau recherche, publiés ou non, émanant des établissements d'enseignement et de recherche français ou étrangers, des laboratoires publics ou privés. 


\title{
Kinematics, Workspace and Singularity Analysis of a Parallel Robot with Five Operation Modes*
}

\author{
Damien Chablat \\ Laboratoire des Sciences du Numérique \\ de Nantes, UMR CNRS 6004 \\ 1 rue de la Noë, 44321 Nantes, France \\ email: damien.chablat@cnrs.fr
}

\author{
Xianwen Kong \\ School of Engineering and Physical \\ Sciences, Heriot-Watt University \\ Edinburgh, EH14 4AS, UK \\ email: X.Kong@hw.ac.uk
}

\author{
Chengwei Zhang \\ School of Engineering and Physical \\ Sciences, Heriot-Watt University \\ Edinburgh, EH14 4AS, UK \\ email: moonlight1780@163.com
}

\begin{abstract}
Most multi-mode parallel robots can change operation modes by passing through constraint singularities. This paper deals with a comprehensive kinematic study of a 3-DOF multi-mode 3-PRPiR parallel robot developed at Heriot-watt University. This robot is able to reach several operation modes without crossing any constraint singularity by using lockable Pi and $R$ joints. Here a Pi joint may act as a 1-DOF planar parallelogram if its lockable $P$ (prismatic) joint is locked or a 2-DOF RR serial chain if its lockable $P$ joint is released. The operation modes of the robot include a $3 T$ operation mode and four $2 T 1 R$ operation modes with two different directions of the rotation axis of the moving platform. The inverse kinematics and forward kinematics of the robot in each operation mode are dealt with in detail. The joint space and workspace analysis of the robot allow us to know the regions of the workspace that the robot can reach in each operation mode. It is shown that the robot is able to change assembly mode in one operation mode by passing through another operation mode.
\end{abstract}

\section{Introduction}

Reconfigurable Parallel Manipulators (PMs), such as multi-mode PMs [1-12], have received much attention from a number of researchers in the past decade [13]. The main characteristics of multi-mode PMs include [7-9]: a) fewer actuators are needed for the moving platform to realize several specified motion patterns; and b) less time is needed in reconfiguring the PM since there is no need to disassemble the PM in the process of reconfiguration.

A number of interesting multi-mode PMs have been proposed, such as multi-mode PMs with a 3-DOF (degrees-offreedom) translational mode and a 3-DOF planar mode or a 4-DOF 3T1R mode [7,8], PMs with two 3T1R (also Schönflies motion) operation mode which has three translational DOF and one rotational DOF operation modes [4], PMs with two 2T1R operation modes [5, 6], and 2-DOF 3-4R PMs with both spherical translation mode and sphere-on-sphere rolling mode [9]. References $[14,15]$ show 4-DOF 3T1R PMs that have an extra 2-DOF or 3-DOF motion mode in addition to the required 4-DOF 3T1R motion mode. It is noted the metamorphic PMs based on reconfigurable $\mathrm{U}$ (universal) or S (spherical) joints $[16,17]$ and the multi-mode PMs with lockable joints $[18,19]$ are in fact kinematically redundant PMs in nature and do not belong to multi-mode PMs that this paper focuses on. In addition, several methods have been proposed [14, 15, 19-25] to the reconfiguration analysis of multi-mode PMs for identifying all the operation modes and the transition configurations of a PM.

\footnotetext{
${ }^{*}$ The original version of this paper has been accepted for presentation at the ASME 2017 International Design Engineering Technical Conferences \& Computers and Information in Engineering Conference, DETC2017-67284, August 6-9, 2017, Cleveland, OH, USA

${ }^{\dagger}$ Currently at Dalian Huarui Heavy Industry Group Co.,Ltd, China
} 
Table 1. Five operation modes of the multi-mode parallel robot.

\begin{tabular}{|c|c|c|c|c|}
\hline \multicolumn{3}{|c|}{ Operation modes } & \multicolumn{2}{c|}{ Conditions } \\
\hline No & $\begin{array}{c}\text { Translation } \\
\text { along }\end{array}$ & $\begin{array}{c}\text { Rotation } \\
\text { about }\end{array}$ & $\begin{array}{c}\text { Joints } \\
\text { locked }\end{array}$ & $\begin{array}{c}\text { Joints } \\
\text { released }\end{array}$ \\
\hline 1 & $x, y, z$ & -- & $P_{1}, P_{2}$ & $R_{1}, R_{2}, R_{3}$ \\
\hline 2 & $x, y$ & $z(\alpha)$ & $R_{1}, R_{2}, P_{2}$ & $R_{3}, P_{1}$ \\
\hline 3 & $x, y$ & $x(\beta)$ & $R_{1}, R_{2}, P_{1}$ & $R_{3}, P_{2}$ \\
\hline 4 & $y, z$ & $x(\beta)$ & $R_{3}, P_{1}$ & $R_{1}, R_{2}, P_{2}$ \\
\hline 5 & $y, z$ & $z(\alpha)$ & $R_{3}, P_{2}$ & $R_{1}, R_{2}, P_{1}$ \\
\hline
\end{tabular}

However, only a few multi-mode PMs, such as the PM with two 1T1R operation modes used as a novel swivel head for machine tools [2], have been put into use in practice. As pointed out in [22,23], one of the causes is the lack of practical methods for switching a PM from one operation mode to another.

In [26], a multi-mode PM was proposed, which is a revised version of the multi-mode PM developed at Heriot-Watt University as shown in Fig. 1 of [18]. This multi-mode PM can switch from one operation mode to another securely by using reconfigurable planar parallelograms and lockable $\mathrm{R}$ (revolute) joints. Unlike robot architectures that cross a constraint singularity to change operation mode, no external force or torque is required to change operation modes. This paper will perform a systematic study on the inverse kinematics, forward kinematics, workspace and singularity analysis of this multimode PM.

In the literature, trajectories for changing assembly modes has been investigated either by using dynamic proper trajectories [27] or the properties of the cuspidal robots [28,29]. In this paper, we will present a new way to change assembly modes, which are associated with different solutions of the forward kinematics, by switching operation modes.

In Section 2, the description of a 3-PRPiR PM is given. Section 3 deals with the inverse and direct kinematic problem. In Section 4, the singularity analysis is presented. Section 5 presents the workspace analysis to find out the connected regions reachable for a given working mode, i.e. a given solution of the inverse kinematics [30], in each operation mode. Finally, conclusions are drawn on the advantages of this robot.

\section{Description of a 3-PRPiR parallel robot}

The 3-DOF 3-PRPiR ${ }^{1}$ multi-mode parallel robot developed by the second author's team at Heriot-Watt University [26] is composed of a base and moving platform connected by three RPiR legs. Here R and Pi represent revolute and reconfigurable parallelogram joint respectively (Fig. 1). The Pi joint may act as a 1-DOF planar parallelogram if its lockable P (prismatic) joint is locked or a 2-DOF RR serial chain if its lockable $\mathrm{P}$ joint is released. In total, there are three lockable revolute joints $\left(R_{1}, R_{2}\right.$ and $\left.R_{3}\right)$ and two lockable prismatic joints $\left(P_{1}\right.$ and $\left.P_{2}\right)$ which can be locked/released to change the operation modes of the robot. With this prototype, it was possible to validate the movements of the robot according to the operation mode. The change of operating mode is carried out in the home pose shown in Fig. 1 or whenever the robot is under the $x y$ or $y z$ planes passing through this pose with $\alpha=0$ and $\beta=0$. A practical home configuration would be one does not encounter parallel singularity. Currently, the change of operation mode is done by inserting pins manually to lock or unlock the joints. In the future, magnetic devices will be used to make this change. Compared to the use of motors, this solution is simpler because a logic output of the controller is used and there is no optical encoder and no drive.

In the home pose, the axes of all the revolute joints, including those within the Pi joint, are parallel to two directions. The input joint variables are the three prismatic actuated joints $\rho_{1}, \rho_{2}$ and $\rho_{3}$ along the $y$-axis. The motion of the moving platform depends on the operation mode of the robot (Fig. 1). Table 1 describes the robot's mobility in its five operation modes.

In a fixed reference frame, the positions of the fixed points $A_{i}(i=1,2,3)$ as well as the points $B_{i}$ controlled by the

\footnotetext{
${ }^{1}$ One of the Pi joint is replaced with an RR serial chain to simplify the structure without affecting the function of the robot.
} 


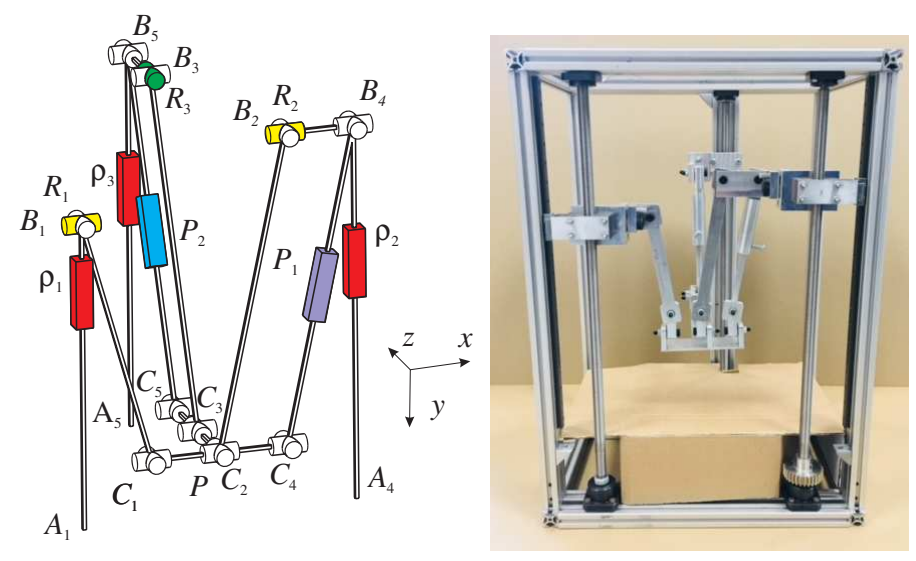

Fig. 1. A 3-PRPiR multi-mode parallel robot with the three actuators in red, the passive joints in white and the lockable joints in other colors and its prototype

actuated prismatic joints are defined as

$$
\begin{aligned}
& A_{1}=\left[-d_{1}, 0,0\right]^{T} \\
& A_{2}=\left[d_{6}, 0,0\right]^{T} \\
& A_{3}=\left[0,0, d_{2}\right]^{T} \\
& A_{4}=\left[d_{6}+d_{7}, 0,0\right]^{T} \\
& A_{5}=\left[0,0, d_{2}+d_{5}\right]^{T}
\end{aligned}
$$

Points $A_{2}$ and $A_{3}$ are virtual points only to simplify the model.

$$
\begin{aligned}
& B_{1}=\left[-d_{1}, \rho_{1}, 0\right]^{T} \\
& B_{2}=\left[d_{6}, \rho_{2}, 0\right]^{T} \\
& B_{3}=\left[0, \rho_{3}, d_{2}\right]^{T} \\
& B_{4}=\left[d_{6}+d_{7}, \rho_{2}, 0\right]^{T} \\
& B_{5}=\left[0, \rho_{3}, d_{2}+d_{5}\right]^{T}
\end{aligned}
$$

The motion of the end-effector $P$ depends on the operation modes of the robot. The coordinates of point $P$ will be given in details in the next subsections on the constraint equations for each operation mode. The rotations around $x$-axis and $z$-axis are denoted by $\beta$ and $\alpha$ respectively.

Based on the prototype built at Heriot-Watt University, the design parameters are defined in millimeters as (Fig. 2): $d_{1}=55, d_{2}=113, d_{3}=385 / 10, d_{4}=95, d_{5}=25, d_{6}=33, d_{7}=405 / 10, l_{1}=124, l_{2}=165$, and $l_{3}=170$. For all the operation mode, the constraint equations are obtained by calculating the distances between the points $B_{i}$ and $C_{i}$.

$$
\left\|B_{i}-C_{i}\right\|=l_{i} \quad \text { for } \mathrm{i}=1,2,3
$$

In the following equations, we will introduce the position and orientation of the end-effector $P$ as a function of the operation modes of the robot.

\section{Operation Mode 1}

Operation mode 1 is a 3-DOF pure translation mode. That is to say the moving platform cannot rotate. This architecture is identical to the linear Delta robot found in the Renault Automation UraneSX [31] or the IRCCyN Orthoglide [32, 33].

$$
\begin{aligned}
& C_{1}=\left[x-d_{3}, y, z\right]^{T} \\
& C_{2}=P=[x, y, z]^{T} \\
& C_{4}=\left[x+d_{7}, y, z\right]^{T} \\
& C_{3}=\left[x, y, z+d_{4}\right]^{T} \\
& C_{5}=\left[x, y, z+d_{4}+d_{5}\right]^{T}
\end{aligned}
$$




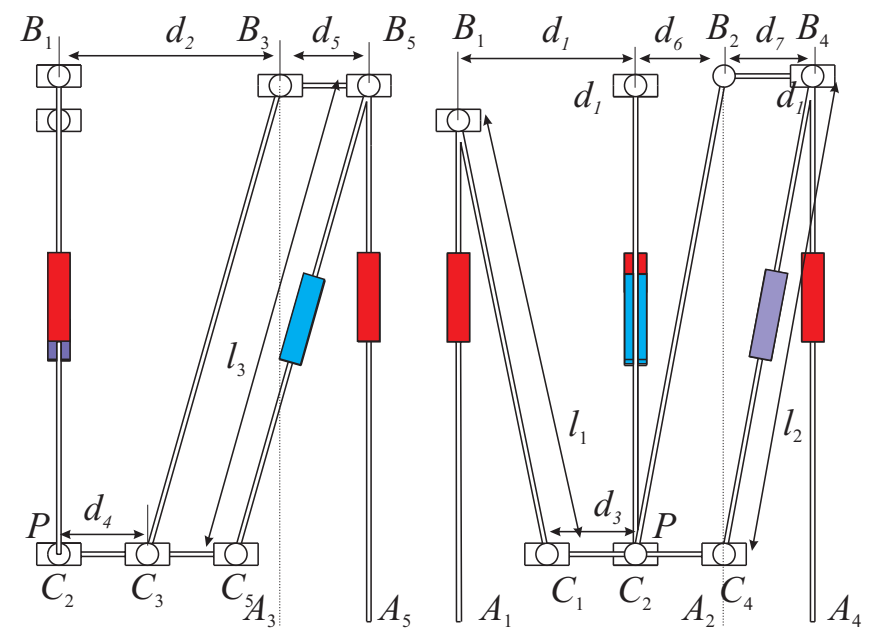

Fig. 2. Link parameters of the 3-PRPiR multi-mode parallel robot.

The constraint equations are

$$
\left\{\begin{array}{l}
\left(x-d_{3}+d_{1}\right)^{2}+\left(y-\rho_{1}\right)^{2}+z^{2}=l_{1}^{2} \\
\left(x-d_{6}\right)^{2}+\left(y-\rho_{2}\right)^{2}+z^{2}=l_{2}^{2} \\
x^{2}+\left(y-\rho_{3}\right)^{2}+\left(z+d_{4}-d_{2}\right)^{2}=l_{3}{ }^{2}
\end{array}\right.
$$

\section{Operation Mode 2}

For this operation mode, the moving platform undergoes a 3-DOF planar motion along the O-XY plane: it translates in the plane $(x, y)$ and rotate around the $z$-axis. We can write the positions of $C_{i}$ and $P$ as

$$
\begin{aligned}
& C_{1}=\left[\begin{array}{ll}
x-d_{3} \cos \alpha, & y-d_{3} \sin \alpha, 0
\end{array}\right]^{T} \\
& C_{2}=P=[x, y, 0]^{T} \\
& C_{3}=\left[x, y, d_{4}\right]^{T} \\
& C_{4}=\left[x+d_{7} \cos \alpha, y+d_{7} \sin \alpha, 0\right]^{T} \\
& C_{5}=\left[x, y, d_{4}+d_{5}\right]^{T}
\end{aligned}
$$

The constraint equations are

$$
\left\{\begin{array}{l}
\left(x-d_{3} \cos \alpha+d_{1}\right)^{2}+\left(y-d_{3} \sin \alpha-\rho_{1}\right)^{2}=l_{1}^{2} \\
\left(x-d_{6}\right)^{2}+\left(y-\rho_{2}\right)^{2}=l_{2}^{2} \\
x^{2}+\left(y-\rho_{3}\right)^{2}+\left(d_{4}-d_{2}\right)^{2}=l_{3}^{2}
\end{array}\right.
$$

\section{Operation Mode 3}

For this operation mode, the translations of the moving platform are in the plane $(x, y)$ and the rotation around the $x$-axis. Such motion is called PPR equivalent motion in [34]. We can write the positions of $C_{i}$ and $P$ as

$$
\begin{aligned}
& C_{1}=\left[x-d_{3}, y, 0\right]^{T} \\
& C_{2}=P=[x, y, 0]^{T} \\
& C_{4}=\left[x+d_{7}, y, 0\right]^{T}
\end{aligned}
$$

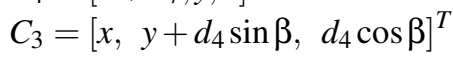

$$
\begin{aligned}
& C 5=\left[x, y+\left(d_{4}+d_{5}\right) \sin \beta,\left(d_{4}+d_{5}\right) \cos \beta\right]^{T}
\end{aligned}
$$

The constraint equations are

$$
\left\{\begin{array}{l}
\left(x-d_{3}+d_{1}\right)^{2}+\left(y-\rho_{1}\right)^{2}=l_{1}^{2} \\
\left(x-d_{6}\right)^{2}+\left(y-\rho_{2}\right)^{2}=l_{2}^{2} \\
x^{2}+\left(y+d_{4} \sin \beta-\rho_{3}\right)^{2}+\left(d_{4} \cos \beta-d_{2}\right)^{2}=l_{3}^{2}
\end{array}\right.
$$




\section{Operation Mode 4}

For operation mode 4, the moving platform undergoes a 3-DOF planar motion along the O-YZ plane. The translations of the moving platform are in the plane $(y, z)$ and the rotation around the $x$-axis. The positions of $C_{i}$ and $P$ are

$$
\begin{aligned}
& C_{1}=\left[0-d_{3}, y, z\right]^{T} \\
& C_{2}=P=[0, y, z]^{T} \\
& C_{4}=\left[0+d_{7}, y, z\right]^{T} \\
& C_{3}=\left[0, y+d_{4} \sin \beta, z+d_{4} \cos \beta\right]^{T} \\
& \left.C_{5}=\left[0, y+\left(d_{4}+d_{5}\right) \sin \beta, z+\left(d_{4}+d_{5}\right) \cos \beta\right]\right]^{T}
\end{aligned}
$$

The constraint equations are

$$
\left\{\begin{array}{l}
\left(d_{1}-d_{3}\right)^{2}+\left(y-\rho_{1}\right)^{2}+z^{2}=l_{1}^{2} \\
d_{6}^{2}+\left(y-\rho_{2}\right)^{2}+z^{2}=l_{2}^{2} \\
\left(y+d_{4} \sin \beta-\rho_{3}\right)^{2}+\left(z+d_{4} \cos \beta-d_{2}\right)^{2}=l_{3}^{2}
\end{array}\right.
$$

This operation mode was not presented in [35] because for the design parameters in [35], the robot was singular throughout its workspace.

\section{Operation Mode 5}

For operation mode 5, the moving platform undergoes a 3-DOF planar motion along the O-YZ plane. The translations of the moving platform are in the plane $(y, z)$ and the rotation around the $z$-axis. The positions of $C_{i}$ and $P$ are

$$
\begin{aligned}
& C_{1}=\left[\begin{array}{lll}
0-d_{3} \cos \alpha, & y-d_{3} \sin \alpha, & z
\end{array}\right]^{T} \\
& C_{2}=P=[0, y, z]^{T}
\end{aligned}
$$

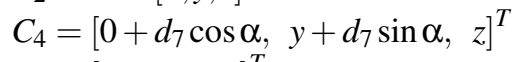

$$
\begin{aligned}
& C_{3}=\left[0, y, z+d_{4}\right]^{T} \\
& C_{5}=\left[0, y, z+d_{4}+d_{5}\right]^{T}
\end{aligned}
$$

The constraint equations are

$$
\left\{\begin{array}{l}
\left(x-d_{3}+d_{1}\right)^{2}+\left(y-\rho_{1}\right)^{2}=l_{1}^{2} \\
\left(x-d_{6}\right)^{2}+\left(y-\rho_{2}\right)^{2}=l_{2}^{2} \\
x^{2}+\left(y+d_{4} \sin \beta-\rho_{3}\right)^{2}+\left(d_{4} \cos \beta-d_{2}\right)^{2}=l_{3}{ }^{2}
\end{array}\right.
$$

\section{Inverse and direct kinematic analysis of the parallel robot in different operation modes}

\subsection{Inverse kinematic analysis}

For each of the five operation modes, there are 8 solutions to the inverse kinematic model or working modes [30]. From the constraint equations [Eqs. (1)-(5)], it is quite straightforward to solve quadratic equations to find the inputs of the actuators. These solutions are given below without detailed derivation.

Figure 3 shows the 8 working modes of the robot for the home pose.

\section{Operation Mode 1}

$$
\begin{aligned}
& \rho_{1}=y \pm \sqrt{2\left(d_{3} d_{1}+x d_{3}-x d_{1}\right)-d_{1}^{2}-d_{3}^{2}+l_{1}^{2}-x^{2}-z^{2}} \\
& \rho_{2}=y \pm \sqrt{2 x d_{6}-d_{6}^{2}+l_{2}^{2}-x^{2}-z^{2}} \\
& \rho_{3}=y \pm \sqrt{2\left(d_{4} d_{2}+z d_{2}-z d_{4}\right)-d_{2}^{2}-d_{4}^{2}+l_{3}^{2}-x^{2}-z^{2}}
\end{aligned}
$$




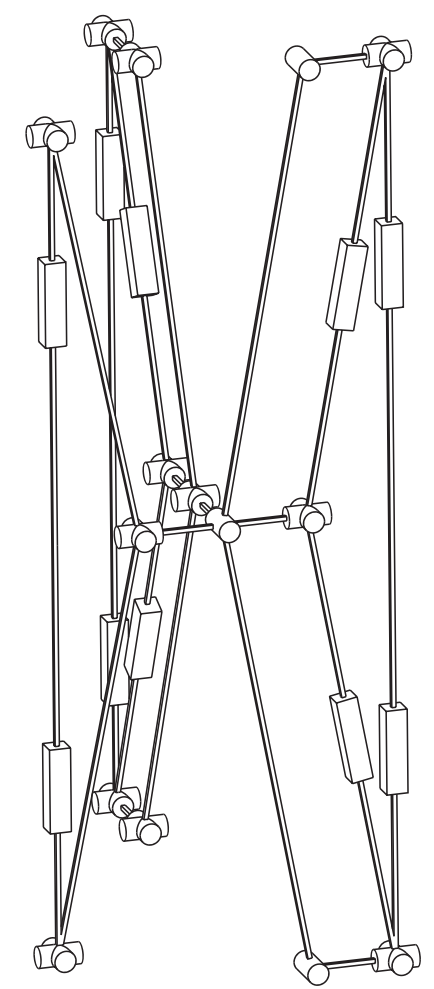

Fig. 3. The eight working modes of the robot associated with the home pose.

\section{Operation Mode 2}

$$
\begin{aligned}
& \rho_{1}=-d_{3} \sin \alpha+y \pm \sqrt{d_{3}^{2} \sin ^{2} \alpha+2\left(d_{3} d_{1}+x d_{3}\right) \cos \alpha-\left(d_{1}+x\right)^{2}-d_{3}^{2}+l_{1}^{2}} \\
& \rho_{2}=y \pm \sqrt{l_{2}^{2}-\left(d_{6}-x\right)^{2}} \\
& \rho_{3}=y \pm \sqrt{l_{3}^{2}-x^{2}-\left(d_{2}-d_{4}\right)^{2}}
\end{aligned}
$$

\section{Operation Mode 3}

$$
\begin{aligned}
& \rho_{1}=y \pm \sqrt{-d_{1}^{2}+2 d_{3} d_{1}-2 x d_{1}-d_{3}^{2}+2 x d_{3}+l_{1}^{2}-x^{2}} \\
& \rho_{2}=y \pm \sqrt{-d_{6}^{2}+2 x d_{6}+l_{2}^{2}-x^{2}} \\
& \rho_{3}=d_{4} \sin \beta+y \pm \sqrt{d_{4}^{2} \sin ^{2} \beta+2 d_{4} \cos \beta d_{2}-d_{2}^{2}-d_{4}^{2}+l_{3}^{2}-x^{2}}
\end{aligned}
$$

\section{Operation Mode 4}

$$
\begin{aligned}
& \rho_{1}=y+\sqrt{l_{1}^{2}-z^{2}-\left(d_{3}-d_{1}\right)^{2}} \\
& \rho_{2}=y+\sqrt{l_{2}^{2}-z^{2}-d_{6}^{2}} \\
& \rho_{3}=d_{4} \sin \beta+y+\sqrt{d_{1}^{2} \sin ^{2} \beta+2 d_{4} \cos \beta\left(d_{2}-z\right)-\left(d_{2}-z\right)^{2}-d_{1}^{2}+l_{3}^{2}}
\end{aligned}
$$




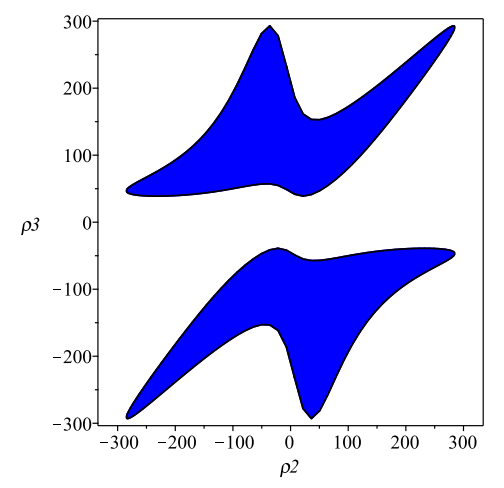

Fig. 4. Joint space of operation mode 1 for $\rho_{1}=0$.

\section{Operation Mode 5}

$$
\begin{aligned}
& \rho_{1}=-d_{3} \sin \alpha+y \pm \sqrt{d_{3}^{2} \sin ^{2} \alpha+2 d_{3} \cos \alpha d_{1}-d_{1}^{2}-d_{3}^{2}+l_{1}^{2}-z^{2}} \\
& \rho_{2}=y \pm \sqrt{l_{2}^{2}-d_{6}^{2}-z^{2}} \\
& \rho_{3}=y \pm \sqrt{2 z d_{2}-\left(d_{2}-d_{4}\right)^{2}-2 d_{4}+l_{3}^{2}-z^{2}}
\end{aligned}
$$

\subsection{Direct kinematic analysis}

Solving the constraint equations associated with each operation mode [Eqs. (1)-(5)], one can find the locations of the moving platform for a given set of inputs.

For operation mode 1, the resolution of the direct kinematic analysis amounts to calculating the intersection of three spheres. An efficient geometric method is presented by Pashkevich in [36]. For the other operation modes, we obtain two values for the position according to $x, \mathrm{y}$ or $z$. For a given orientation of the moving platform, we obtain a quadratic equation as a function of the position parameters. Therefore, we usually have four real solutions for the orientation of the moving platform.

Since the equations for all the five operation modes are too large to be displayed in an article and the solutions are well-documented in the literature, we will focus on the variation of the number of solutions to the direct kinematic model in the joint space. For clarity, we make a slice in the joint space by setting $\rho_{1}=0$. This is equivalent to the change of variable $\rho_{2}^{\prime}=\rho_{2}-\rho_{1}$ and $\rho_{3}^{\prime}=\rho_{3}-\rho_{1}$. Figures $4-8$ represent the joint space in blue, the regions where the direct kinematic model admits two real solutions and in red, the regions where it admits four real solutions.

In operation mode 1, there is two separate regions where the direct kinematic problem admits two solutions (Fig. 4).

In operation mode 2, there are eight regions where the direct kinematic problem admits either two or four solutions (Fig. 5) that we can merge in two connected regions. However, there is one hole in each one. This property can cause problems if the robot is to be protected by introducing limits on the active joints.

In operation mode 3, there are three regions that we can merge in a single connected regions (Fig. 6). The region with four direct kinematic solutions connects two regions where there are only two solutions.

In operation mode 4, there are two connected regions (Fig. 7). In each one, we have four regions with either two or four solutions.

In operation mode 5, there are four connected regions with two holes (Fig. 8). The regions with four direct kinematic solutions are inside a region with two solutions.

It is noted that except operation mode 3, the regions in the joint space are not connected. Figures 9-13 shows several postures of the robot when we have the two or four solutions for the direct kinematics in these five operation modes.

\section{Singularity analysis of the parallel robot in different operation modes}

The singularity analysis of conventional parallel robots has been well-documented in the literature. The singularity analysis of a multi-operation mode parallel robot involves the singularity analysis of the robot in each operation mode. In each operation mode, we first find the parallel and serial Jacobian matrix, named $\mathbf{A}$ and $\mathbf{B}$ respectively [37,38], by differentiating the constraint equations [Eas. (1)-(5)] with resnect to time. Then we obtain the narallel and serial singularities by studving 


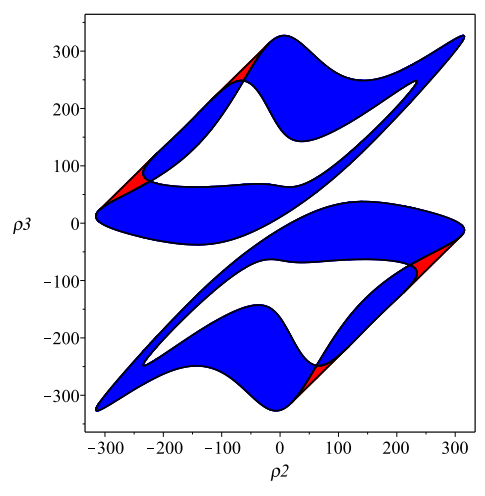

Fig. 5. Joint space of operation mode 2 for $\rho_{1}=0$.

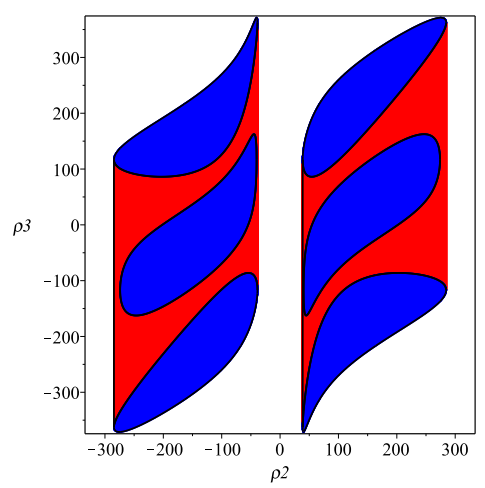

Fig. 7. Joint space of operation mode 4 for $\rho_{1}=0$.

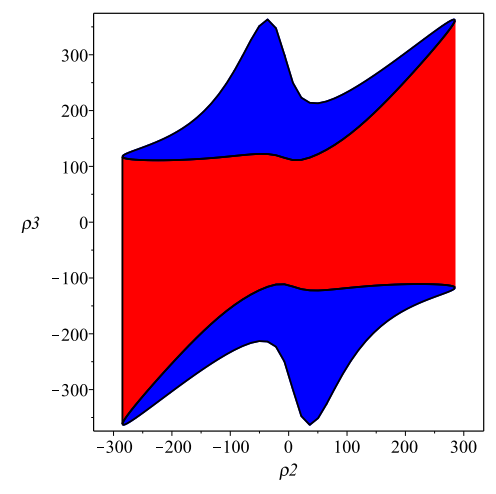

Fig. 6. Joint space of operation mode 3 for $\rho_{1}=0$.

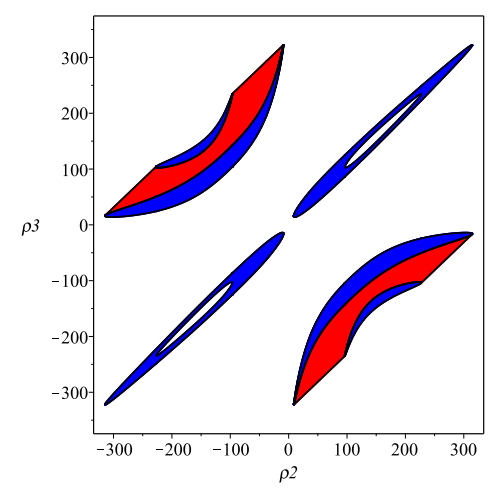

Fig. 8. Joint space of operation mode 5 for $\rho_{1}=0$.
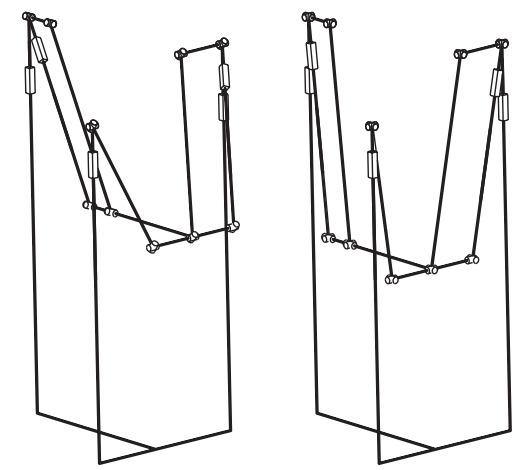

Fig. 9. Example of joint configuration with two direct kinematic solutions in operation mode 1 with $\rho_{1}=-50, \rho_{2}=0$, and $\rho_{3}=0$.

$\operatorname{det}(\mathbf{A})$ and $\operatorname{det}(\mathbf{B})$ respectively [30]. In each operation mode, the serial singularities are located at the boundaries of the workspace while the parallel singularities divide it.

As the robots studied have several solutions to the direct and inverse kinematic problem, the singularity conditions are defined in the cross product of the joint space and workspace. Their projections into the workspace are not requested because we cannot distinguish the curves associated with a given working mode. The equations of the singularities are written according to the design parameters of the prototype because it is not possible to write these conditions in this paper without affecting their values. However, the Siropa library makes it possible to do all the calculations in the general case with all the design parameters $\left(d_{i}\right.$ for $i=1 . .7$ and $l_{i}$ for $i=1,2,3$ [39]. For example, for the operation mode 1 , without assigning the design parameters, the number of operands of the parallel singularity locus in the Cartesian space is 2929 and the degree of the nolvnomial is 12 

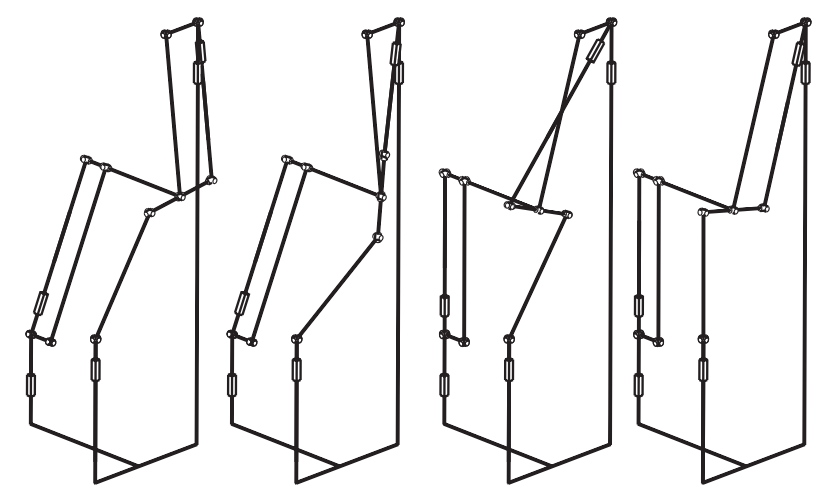

Fig. 10. Example of joint configuration with four direct kinematic solutions in operation mode 2 with $\rho_{1}=0, \rho_{2}=272$, and $\rho_{3}=-53$.
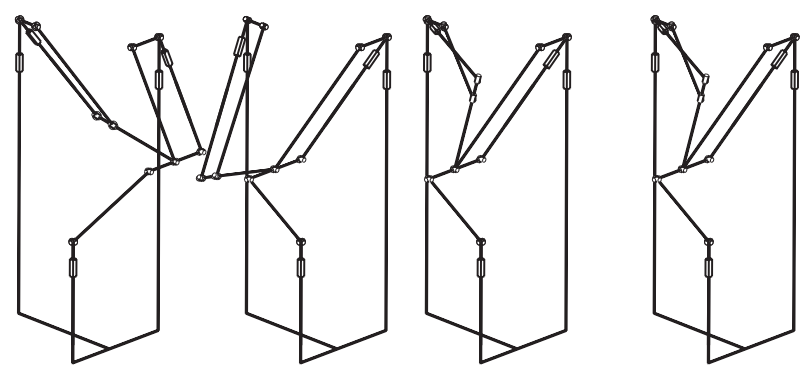

Fig. 11. Example of joint configuration with four direct kinematic solutions in operation mode 3 with $\rho 1=0, \rho_{2}=200$, and $\rho_{3}=200$.
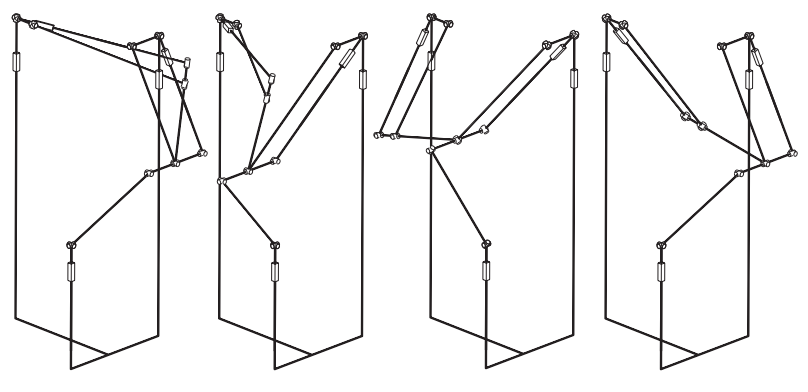

Fig. 12. Example of joint configuration with four direct kinematic solutions in operation mode 4 with $\rho 1=0, \rho_{2}=200$, and $\rho_{3}=200$.

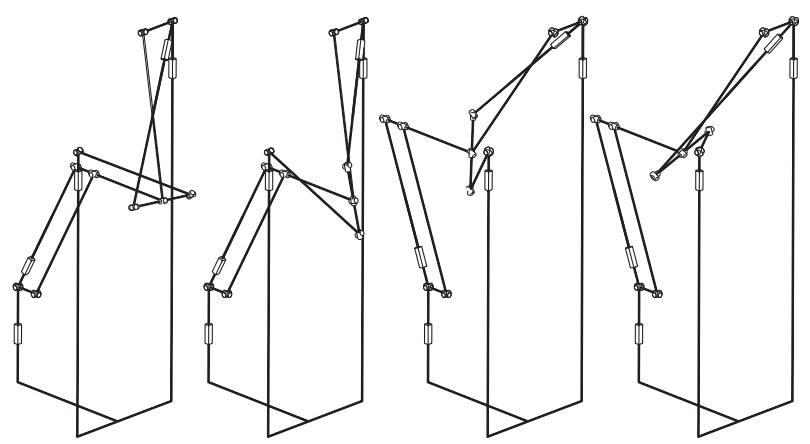

Fig. 13. Example of joint configuration with four direct kinematic solutions in operation mode 5 with $\rho 1=0, \rho_{2}=100$, and $\rho_{3}=-200$.

\subsection{Operation mode 1}

The singular configurations in operation mode 1 of this robot are the same as the conventional parallel robot presented in [40]. We have the serial singularities when the legs are orthogonal to the actuators and the narallel singularities when the 
legs are in the same plane (flat position) or all parallel (bar position).

Serial singularities occur if and only if

$$
\rho_{3}-y=0, \rho_{2}-y=0 \text { or } \rho_{1}-y=0
$$

Parallel Singularities occur if and only if

$$
(-12 x-22 z+396) \rho_{1}+(12 x-11 z+198) \rho_{2}+33 z \rho_{3}-594 y=0
$$

In this operation mode, the determinant of the matrix $\mathbf{A}$ cannot be factorized. In a single equation, we have the singularity locus associated with the eight working modes.

\subsection{Operation mode 2}

Serial singularities occur if and only if

$$
77 \sin \alpha-2 y+2 \rho_{1}=0, \rho_{2}-y=0 \text { or } \rho_{3}-y=0
$$

Only the serial singularities associated with the first leg $\rho_{1}$ depend on the orientation of the moving platform.

Parallel Singularities occur if and only if

$$
x \sin \alpha+\cos \alpha \rho_{1}-y \cos \alpha+55 \sin \alpha=0 \quad \text { or } \quad x \rho_{2}-\rho_{3} x+33 \rho_{3}-33 y=0
$$

The determinant of the matrix $\mathbf{A}$ is factorized into two components. This properties means that the workspace is divided into at least four regions. We notice that the first component does not depend on the orientation of the moving platform.

\subsection{Operation mode 3}

Serial singularities occur if and only if

$$
\rho_{1}-y=0 \text { or } \rho_{2}-y=0 \text { or } y+95 \sin \beta-\rho_{3}=0
$$

As the axis of rotation of the moving platform is parallel to the $x$-axis, only the serial singularities related to the third leg depend on the orientation of the moving platform.

Parallel Singularities occur if and only if

$$
\cos \beta\left(\rho_{3}-y\right)-113 \sin \beta=0 \quad \text { or } \quad 2 x\left(\rho_{1} x-\rho_{2}\right)-66 \rho_{1}-33 \rho_{2}+99 y=0
$$

\subsection{Operation mode 4}

Serial singularities occur if and only if

$$
\rho_{1}-y=0 \text { or } \rho_{2}-y=0 \text { or } y+95 \sin \beta-\rho_{3}=0
$$

As the axis of rotation of the moving platform is parallel to the $x$-axis, only the serial singularities related to the first leg depend on the orientation of the moving platform.

Parallel Singularities occur if and only if

$$
\cos \beta \rho_{3}-y \cos \beta+z \sin \beta-113 \sin \beta=0 \quad \text { or } \quad z=0 \quad \text { or } \quad \rho_{1}-\rho_{2}=0
$$




\subsection{Operation mode 5}

Serial singularities occur if and only if

$$
77 \sin \alpha+2\left(\rho_{1}-y\right)=0 \text { or } \rho_{2}-y=0 \text { or } \rho_{3}-y=0
$$

As the axis of rotation of the moving platform is parallel to the $z$-axis, only the serial singularities related to the first leg depend on the orientation of the moving platform.

Parallel Singularities occur if and only if

$$
\cos \alpha \rho_{1}-y \cos \alpha+55 \sin \alpha \quad \text { or } \rho_{2} z-z \rho_{3}-18 \rho_{2}+18 y=0
$$

\section{Workspace analysis of the parallel robot in different operation modes}

\subsection{Definition of the aspects}

The workspace analysis of a multi-mode parallel robot requires analyzing the workspace of the robot in each operation mode. In each operation mode, one needs to separate the postures of the robot according to the current working modes. An aspect was defined in [30] as the maximal sets in the product of the workspace, $W$, by the joint space, $Q$. However, we can only represent its projection in the workspace that we will call parallel aspects, for a given working mode.

Since that it is impossible to change the working modes without disassembling this multi-mode parallel robot, we will study only the working modes depicted in Fig. 1 for simplicity reasons. With the Siropa library, we can make an algebraic cylindrical decomposition (CAD) $[41,42]$ in the space which includes the joint space and the workspace taking into account the serial and parallel singularities. In this paper, we assume there is no limitation on the range of motion of the joints. Therefore, we can study the workspace by considering only its section at $y=0$.

In each cross-section of the workspace, we have represented the parallel aspects [30], i.e., the largest regions without singularity in the Cartesian space. The boundaries of these regions are the parallel singularities. Curves may exist that do not divide the workspace. These curves are associated with the other operation modes. These curves are obtained by the discriminant varieties of the constraint equations with the serial and parallel singularities [43].

\subsection{Workspace analysis}

In operation mode 1, the workspace is a divided in two regions due to the parallel singularities. In Fig. 14, the curves correspond to the parallel singularities associated with the 8 working modes and only a part of these curves divides the workspace in to two regions for the working mode under study. Unlike parallel robots with equal lengths and three parallel actuators [35], one singularity curve is associated to the working mode of the Fig. 1. In operation mode 2, the workspace is divided in two regions (Fig. 15). Please note the continuity of certain regions due to the cyclicity of the angles.

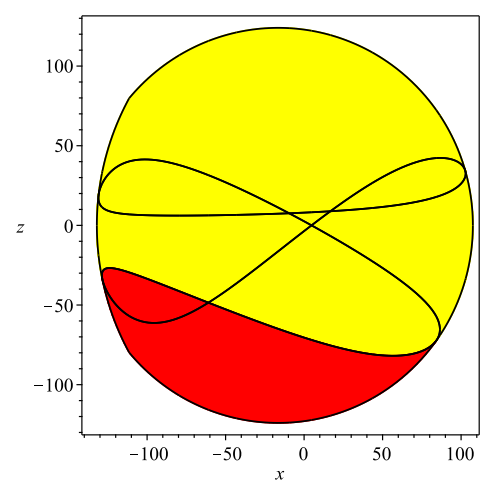

Fig. 14. Workspace in operation mode 1 for $y=0$.

The workspace in operation mode 3 consists of only two regions (Fig. 16). The workspace in operation mode 4 has four aspects (Fig. 17). The brown region is connected due to the cyclicity of the angles. For $z=0$, and for any orientation, the robot is in singularity.

The workspace in operation mode 5 has two aspects (Fig. 18). The green region is connected due to the cyclicity of the 


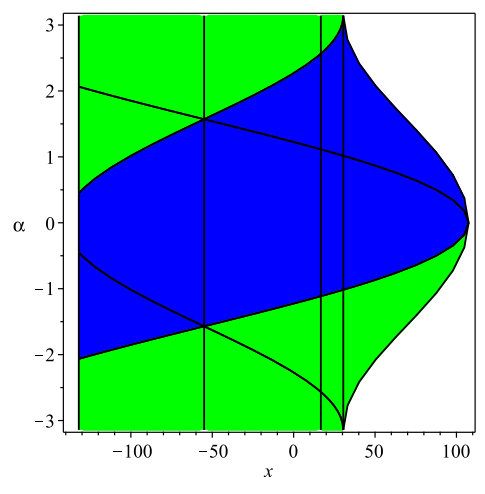

Fig. 15. Workspace in operation mode 2 for $y=0$.

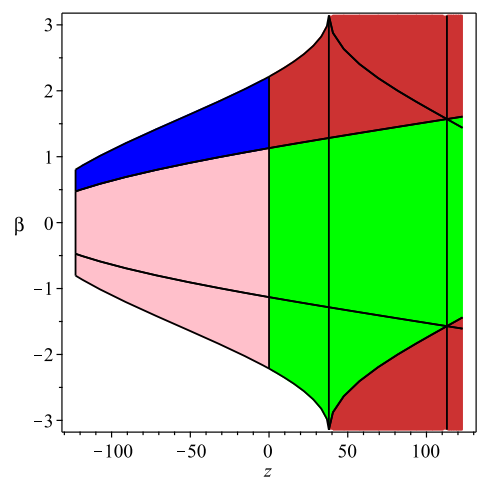

Fig. 17. Workspace in operation mode 4 for $y=0$.

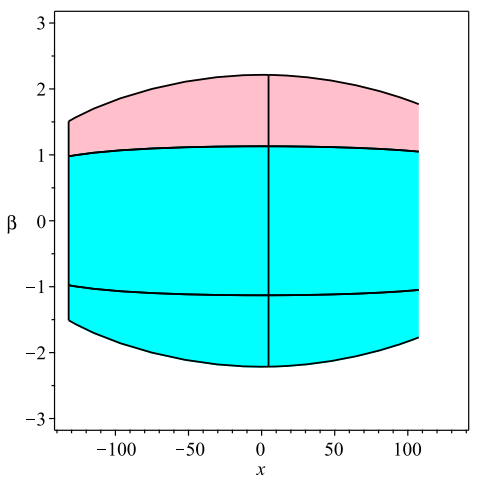

Fig. 16. Workspace in operation mode 3 for $y=0$.

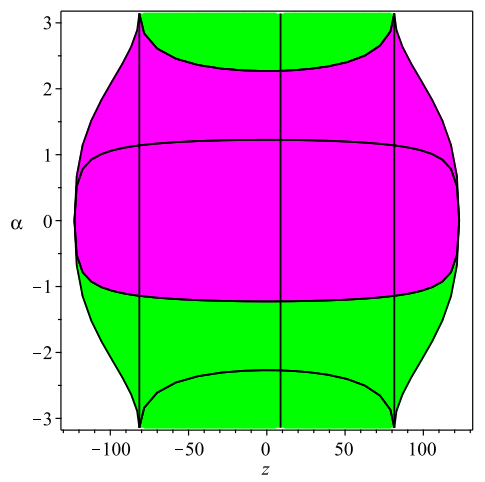

Fig. 18. Workspace in operation mode 5 for $y=0$.

\subsection{Connectivity analysis}

The multi-mode parallel robot can switch among its five operation modes in its home pose in which $x=0, z=0, \alpha=0$ and $\beta=0$. In Fig. 19, starting from the home pose, the red arrows show the corresponding postures for each operation mode. The grey regions are the regions of the workspace that the robot cannot reach without crossing a parallel singularity.

Based on this analysis, Figs. 20 and 21 depict, at the left side, the workspace for the operations modes 1, 2 and 5 and the operation modes 1,3 and 4 , respectively, and at the right side, the remaining connected regions.

In operation mode 1 , only the yellow region can be reached if we do not switch to operation modes 4 or 5 . For example, in the operation mode 1, the trajectory $T_{1}$ to $T_{2}$ defined in Fig. 22(a) is not feasible because the point $T_{3}$ is singular. So, along the negative $z$-axis, we can reach at most $z=-70.456$ for $x=0$ starting from the home pose. In operation mode 4 , the robot is singular at any configuration with $z=0$ (Fig. 22(c)). The pink and green regions can be reached only if we apply a trajectory either with operation mode 1 or operation mode 5 as shown Fig. 21. Conversely, in the operation mode 5 (Fig. 22(b)), the trajectory $T_{1}$ to $T_{2}$ is fully included inside a single region.

Based on the above results, we conclude that it is possible to change the parallel aspects for a given operation mode by passing through operation mode 1 or 4 or 5 (Figs. 22(a)-(c)). This change only happens at configurations with $x=0$ and any values of $y$ (Fig. 23). This property increases the workspace of the robot theoretically.

We notice that the ranges of translation in operation modes 2, 3, 4 and 5 are within the ranges defined by the intersections of the workspace with the $x$ - and $z$-axes in operation mode 1. The above analysis has shown that the asymmetric 3-PRPiR multi-mode PM is better than the symmetric case of the multi-mode PM detailed in [35].

\section{Conclusions}

In this paper, we have presented the kinematics of a multi-mode parallel robot that can change operation modes by using reconfigurable Pi joints and lockable $\mathrm{R}$ joints without the problem of the constraint singularities. Changes in operation modes are realized by locking/releasing certain lockable joints. This feature allow us to know the operation mode without external or internal sensors. We have investigated the singularities of the parallel robot in different operation modes from the study of the determinant of the Jacobian matrices. For an operation mode resulting from the "home" configuration of the robot, we have decomnosed the worksnace into asnects and renresented their nroiection in the worksnace. The conditions for switching from 


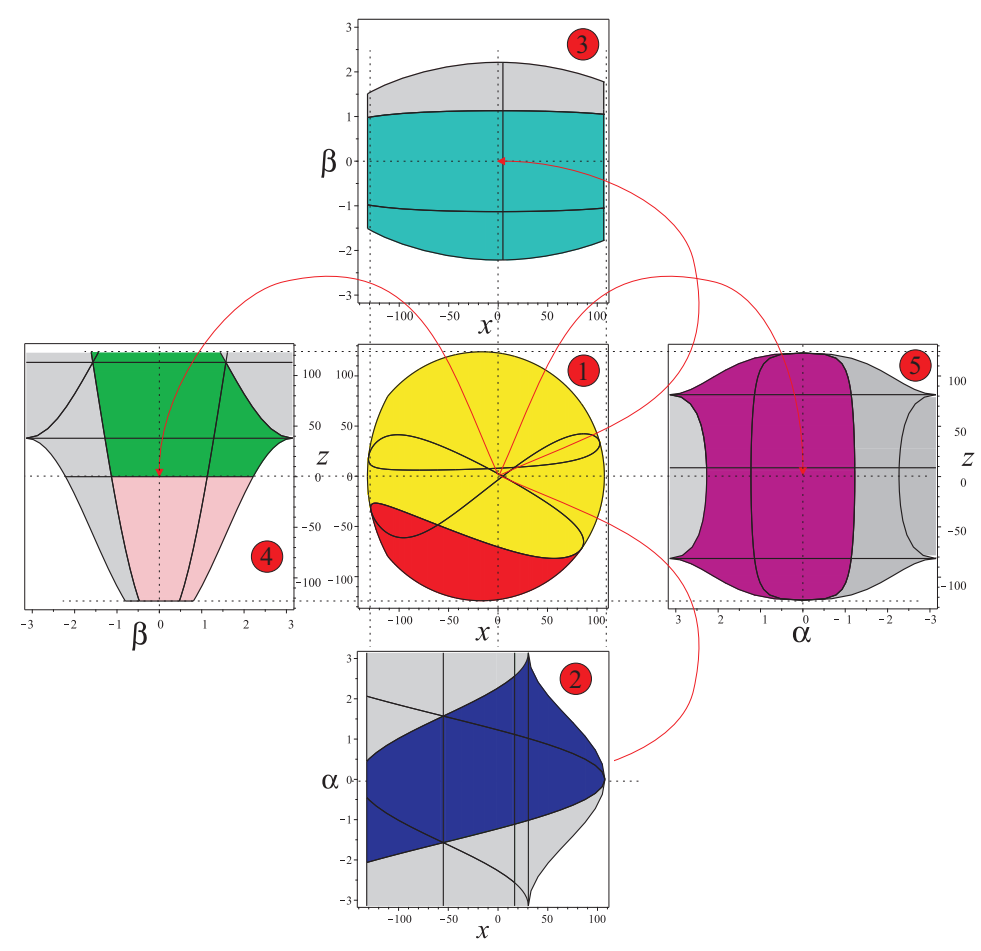

Fig. 19. Transition among the five operation modes.

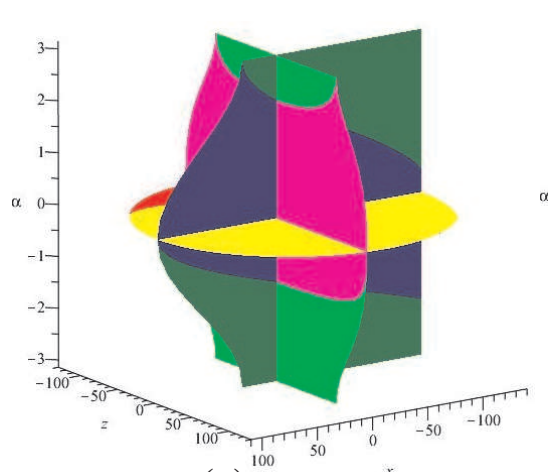

(a)

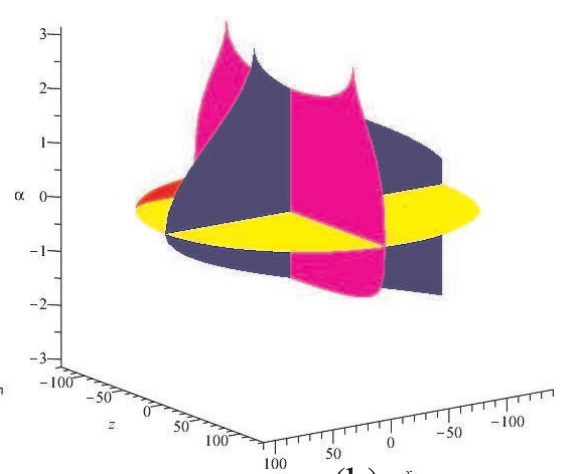

(b)

Fig. 20. Workspace (a) and connected regions (b) for $y=0$ for translation motion and the rotations around the $z$-axis

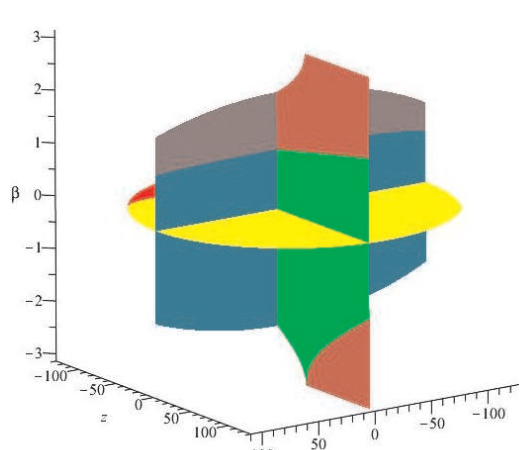

(a)

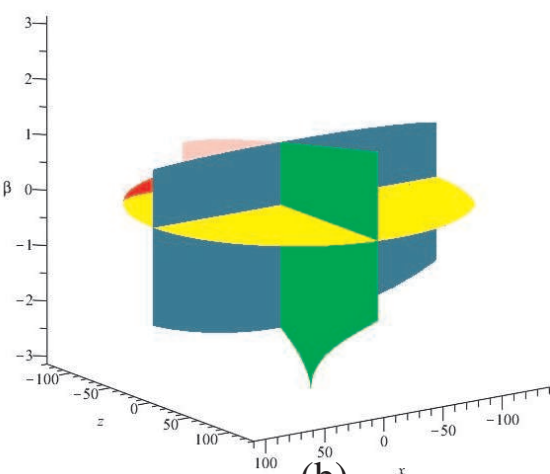

(b)

Fig. 21. Workspace (a) and connected regions (b) for $y=0$ for translation motion and the rotations around the $x$-axis 


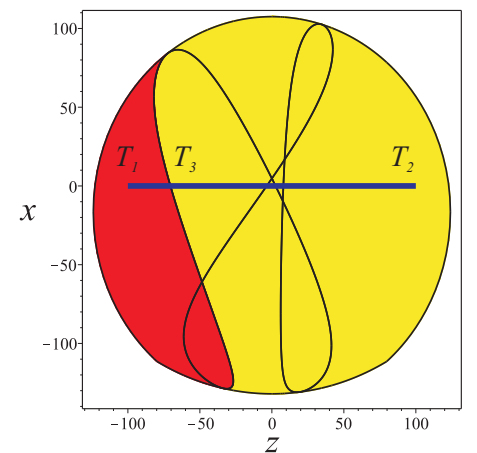

(a)

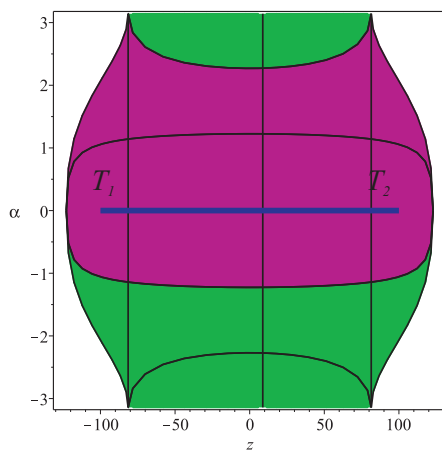

(b)

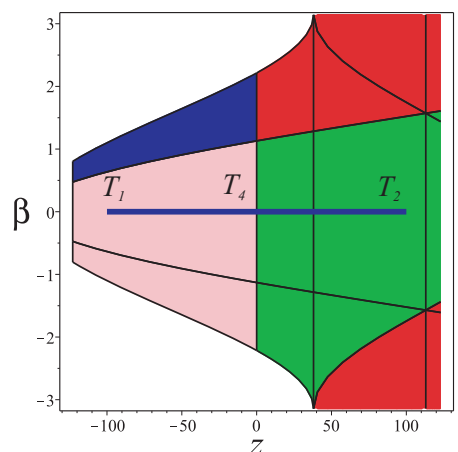

(c)

Fig. 22. Non-singular and singular assembly mode changing trajectory for three operation modes for $T_{1}=[-100,0], T_{2}=[100,0]$, $T_{3}=[-70.456,0]$ and $T_{4}=[0,0]$ where $x=0$ in (b) and (c), $\beta=0$ in (a) and (b), $\alpha=0$ in (a) and (c).

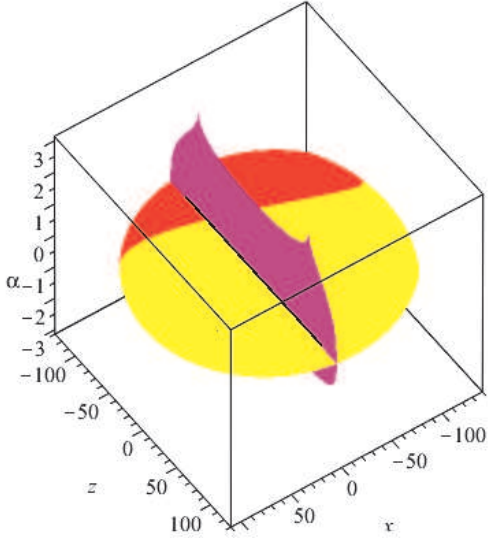

(a)

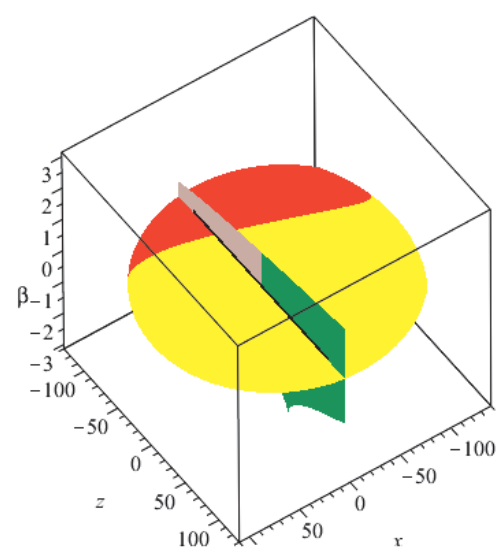

(b)

Fig. 23. Non-singular and singular assembly mode changing trajectory for $\beta=0$ (a) and $\alpha=0$ (b) between $T_{1}=[-100,0]$ and $T_{2}=$ $[100,0]$.

one operation mode to another have been identified. The aspects that the robot can achieve in a given operation mode without going through a parallel singularity have also been obtained. Two non-singular assembly mode changing trajectories have been introduced by changing operation mode. This feature can justify the additional brakes requested to change operation mode by increasing the reachable workspace of one operation mode.

This work, together with the literature on the reconfiguration analysis of multi-mode parallel mechanisms [15,20-23], provide a comprehensive framework for the analysis of multi-mode parallel robots.

Future works will be done on the prototype by adding some brakes to automatically change operation mode and optimize the values of the design parameters to optimize the accuracy of the robot as well as its stiffness.

\section{Acknowledgments}

Dr. Xianwen Kong would like to thank the Engineering and Physical Sciences Research Council (EPSRC), United Kingdom, for the support under grant No.EP/K018345/1.

\section{References}

[1] Fanghella, P., Galletti, C., and Gianotti, E., "Parallel robots that change their group of motion," Advances in Robot Kinematics, Springer, The Netherlands, pp. 49-56, 2006.

[2] Refaat, S., Hervé, J.M., Nahavandi, S., and Trinh, H., "Two-mode overconstrained three-DOFs rotational-translational linear-motor-based narallel kinematics mechanism for machine tool annlications." Rohotica 25 nn $461-466.2007$ 
[3] Kong, X., Gosselin, C., and Richard, P.L., "Type synthesis of parallel mechanisms with multiple operation modes," ASME Journal of Mechanical Design, 129(6), pp. 595-601, 2007.

[4] Li, Q. and Hervé, J.M., "Parallel mechanisms with bifurcation of Schoenflies motion," IEEE Transactions on Robotics, 25(1), pp. 158-164, 2009.

[5] Gogu, G., "Maximally regular T2R1-type parallel manipulators with bifurcated spatial motion," ASME Journal of Mechanisms and Robotics, 3(1), 011010, 2011.

[6] Ruggiu, M. and Kong, X., "Mobility and kinematic analysis of a parallel mechanism with both PPR and planar operation modes," Mechanism and Machine Theory, 55, pp. 77-90, 2012.

[7] Kong, X., "Type synthesis of variable degrees-of-freedom parallel manipulators with both planar and 3T1R operation modes," Proceedings of ASME 2012 International Design Engineering Technical Conferences \& Computers and Information in Engineering Conference, DETC2012-70621, Chicago, USA, August 12-15, 2012.

[8] Kong, X., "Type synthesis of 3-DOF parallel manipulators with both a planar operation mode and a spatial translational operation mode," ASME Journal of Mechanisms and Robotics, 5(4): 041015, 2013.

[9] Kong, X., and Yu, J., "Type synthesis of two-degrees-of-freedom 3-4R parallel mechanisms with both spherical translation mode and sphere-on-sphere rolling mode," Journal of Mechanisms and Robotics, 7(4), 041018, 2015.

[10] Gan, D., and Dai, J.S., "Geometry constraint and branch motion evolution of 3-PUP parallel mechanisms with bifurcated motion," Mechanism and Machine Theory, 61, pp. 168-183, 2013.

[11] Zeng, Q., and Ehmann, K.F., "Design of parallel hybrid-loop manipulators with kinematotropic property and deployability," Mechanism and Machine Theory, 71, pp. 1-26, 2014.

[12] Ye, W., Fang, Y., Zhang, K., and Guo, S., "A new family of reconfigurable parallel mechanisms with diamond kinematotropic chain," Mechanism and Machine Theory, 74, pp. 1-9, 2014.

[13] Ding, X., Kong, X., and Dai, J. S., Advances in Reconfigurable Mechanisms and Robots II. Switzerland: Springer International Publishing, 2016.

[14] Coste, M., and Kartoue Mady Demdah, K.M., "Extra modes of operation and self motions in manipulators designed for Schoenflies motion.” Journal of Mechanisms and Robotics,7 (4), 041020, 2015.

[15] Nurahmi, L., Caro, S., Wenger, Ph., Schadlbauer, J., and Husty, M., "Reconfiguration analysis of a 4-RUU parallel manipulator." Mechanism and Machine Theory, 96, pp.269-289, 2016.

[16] Gan, D., Dai, J. S., and Liao, Q., "Mobility change in two types of metamorphic parallel mechanisms," ASME Journal of Mechanisms and Robotics, 1(4), 041007, 2009.

[17] Zhang K. T., Dai J. S., Fang Y. F., "Geometric constraint and mobility variation of two 3-SPS metamorphic parallel mechanisms.” ASME Journal of Mechanisms and Robotics, 135(1): 011001, 2012.

[18] Kong, X., and Jin, Y., "Type synthesis of 3-DOF multi-mode translational/spherical parallel mechanisms with lockable joints," Mechanism and Machine Theory, 96, pp. 323-333, 2015.

[19] Carbonari, L. Callegari, M. Palmieri, G., and Palpacelli, M.-C., A new class of reconfigurable parallel kinematic machines. Mechanism and Machine Theory, 79:pp. 173-183, 2014.

[20] Walter, D.R., Husty, M.L. and Pfurner, M., Chapter A: Complete kinematic analysis of the SNU 3-UPU parallel manipulator, In: Contemporary Mathematics, 496, American Mathematical Society, pp. 331-346, 2009.

[21] Kong, X., "Reconfiguration analysis of a 3-DOF parallel mechanism using Euler parameter quaternions and algebraic geometry method," Mechanism and Machine Theory, 74, pp. 188-201, 2014.

[22] Kong, X., "Reconfiguration analysis of a 4-DOF 3-RER parallel manipulator with equilateral triangular base and moving platform," Mechanism and Machine Theory, 98, pp. 180-189, 2016.

[23] Kong, X., "Reconfiguration analysis of a variable degrees-of-freedom parallel manipulator with both 3-DOF planar and 4-DOF 3T1R operation modes," Proceedings of ASME 2016 International Design Engineering Technical Conferences \& Computers and Information in Engineering Conference, DETC2016-59203, Charlotte, USA, August 21-24, 2016.

[24] Chablat, D., Jha, R., Rouillier, F., Moroz G., "Non-singular assembly mode changing trajectories in the workspace for the 3-RPS parallel robot," Advances in Robot Kinematics, pp. 149-159, 2014.

[25] Chablat, D., Jha, R., Rouillier, F., Moroz G., "Workspace and joint space analysis of the 3-RPS parallel robot," Proceedings of ASME 2014 International Design Engineering Technical Conferences \& Computers and Information in Engineering Conference, Buffalo, USA, August 1720, 2014.

[26] Zhang, C., Improvement of a disassembly-free reconfigurable parallel manipulator, MSc Dissertation, Heriot-Watt University, Edinburgh, UK, 2015.

[27] Nenchez, D. N., Uchiyama M., "Singularity-consistent path tracking: A null space based approach”, IEEE International Conference on Robotics and Automation, pp. 2482-2489, 1995.

[28] Innocenti C., Parenti-Castelli V., "Singularity-free evolution from one configuration to another in serial and fullyparallel manipulators", ASME Journal of Mechanisms Design, 120(1), pp. 73-79, 1998.

[29] Zein M., Wenger P., and Chablat D., "Non-singular assembly-mode changing motions for 3-RPR parallel manipulators", Mechanism and Machine Theory, 43(4), pp. 480-490, 2008.

[30] Chablat $\mathrm{D}$.. Wenger Ph.. "Working modes and aspects in fullv-parallel manipulator." Proceedings of IEEE International 
Conference on Robotics and Automation, pp. 1964-1969, May 1998.

[31] Company, O. and Pierrot, F., "Modelling and design issues of a 3-axis parallel machine-tool," Mechanism and Machine Theory, 37, pp. 1325-1345, 2002.

[32] Chablat D. and Wenger Ph., "Architecture optimization of a 3-DOF parallel mechanism for machining applications, the Orthoglide," IEEE Transactions On Robotics and Automation, 19(3), pp. 403-410, June, 2003.

[33] Chablat D., Wenger P., Majou F. and Merlet J.P., "An interval analysis based study for the design and the comparison of 3-DOF parallel kinematic machines,” International Journal of Robotics Research, pp. 615-624, Vol.23(6), June, 2004.

[34] Kong, X. and Gosselin, C.M., "Type synthesis of 3-DOF PPR-equivalent parallel manipulators based on screw theory and the concept of virtual chain," ASME Journal of Mechanical Design, 127(6), 1113-1121.

[35] Chablat D., Kong X., Zhang C., "Kinematics, workspace and singularity analysis of a multi-mode parallel robot", ASME 2017 International Design Engineering Technical Conferences \& Computers and Information in Engineering Conference, Aug 2017, Cleveland, United States. 2017

[36] Pashkevich A, Chablat D. and Wenger Ph., "Kinematics and workspace analysis of a three-axis parallel manipulator: the Orthoglide, Robotica, 24(1), pp. 39-49, 2006.

[37] Gosselin C., and Angeles J., "Singularity analysis of closed-loop kinematic chains," in IEEE Transactions on Robotics and Automation, 6(3), pp. 281-290, June 1990.

[38] Wenger Ph., Chablat D., "Definition sets for the direct kinematics of parallel manipulators," 8th International Conference in Advanced Robotics, pp. 859-864, 1997.

[39] Siropa, "A library for manipulator singularities analysis," accessed January 30, 2018, http://siropa.gforge.inria.fr/doc/files/Siropa/modeling-mpl.html.

[40] Pashkevich A., Wenger Ph., and Chablat D., "Design strategies for the geometric synthesis of Orthoglide-type mechanisms," Mechanism and Machine Theory, 40(8), pp. 907-930, August 2005.

[41] Collins, G. E., Quantifier Elimination for Real Closed Fields by Cylindrical Algebraic Decomposition, Springer Verlag, 1975.

[42] Manubens M., Moroz G., Chablat D., Wenger P, Rouiller F., “Cusp points in the parameter space of degenerate 3-RPR planar parallel manipulators," ASME Journal of Mechanisms and Robotics, 4(4), November, 2012.

[43] Lazard, D. and Rouillier, F., "Solving parametric polynomial systems", Journal of Symbolic Computation, 42(6):636667, 2007. 


\section{List of Figures}

1 A 3-PRPiR multi-mode parallel robot with the three actuators in red, the passive joints in white and the

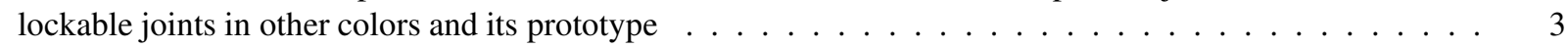

2 Link parameters of the 3-PRPiR multi-mode parallel robot. . . . . . . . . . . . . . . . . . . .

3 The eight working modes of the robot associated with the home pose. . . . . . . . . . . . . . 6

4 Joint space of operation mode 1 for $\rho_{1}=0 . \ldots \ldots \ldots \ldots$

5 Joint space of operation mode 2 for $\rho_{1}=0 . \ldots \ldots \ldots$

6 Joint space of operation mode 3 for $\rho_{1}=0 \ldots \ldots \ldots \ldots$

7 Joint space of operation mode 4 for $\rho_{1}=0 . \ldots \ldots \ldots$

8 Joint space of operation mode 5 for $\rho_{1}=0 . \ldots \ldots \ldots$

9 Example of joint configuration with two direct kinematic solutions in operation mode 1 with $\rho_{1}=-50, \rho_{2}=$ 0 , and $\rho_{3}=0 \ldots \ldots \ldots \ldots \ldots \ldots \ldots \ldots$

10 Example of joint configuration with four direct kinematic solutions in operation mode 2 with $\rho_{1}=0, \rho_{2}=$ 272 , and $\rho_{3}=-53 \ldots \ldots \ldots \ldots \ldots \ldots$

11 Example of joint configuration with four direct kinematic solutions in operation mode 3 with $\rho 1=0, \rho_{2}=$ 200 , and $\rho_{3}=200 \ldots \ldots \ldots \ldots \ldots \ldots \ldots \ldots$

12 Example of joint configuration with four direct kinematic solutions in operation mode 4 with $\rho 1=0, \rho_{2}=$ 200 , and $\rho_{3}=200 \ldots \ldots \ldots \ldots \ldots \ldots \ldots \ldots \ldots$

13 Example of joint configuration with four direct kinematic solutions in operation mode 5 with $\rho 1=0, \rho_{2}=$ 100 , and $\rho_{3}=-200 \ldots \ldots \ldots \ldots \ldots \ldots \ldots$

14 Workspace in operation mode 1 for $y=0 \ldots \ldots \ldots \ldots$

15 Workspace in operation mode 2 for $y=0 . \ldots \ldots \ldots \ldots$

16 Workspace in operation mode 3 for $y=0 . \ldots \ldots \ldots \ldots$

17 Workspace in operation mode 4 for $y=0 . \ldots \ldots \ldots$

18 Workspace in operation mode 5 for $y=0 \ldots \ldots \ldots \ldots$

19 Transition among the five operation modes. . . . . . . . . . . . . . . . . . . . . 13

20 Workspace (a) and connected regions (b) for $y=0$ for translation motion and the rotations around the $z$-axis 13

21 Workspace (a) and connected regions (b) for $y=0$ for translation motion and the rotations around the $x$-axis

22 Non-singular and singular assembly mode changing trajectory for three operation modes for $T_{1}=[-100,0]$, $T_{2}=[100,0], T_{3}=[-70.456,0]$ and $T_{4}=[0,0]$ where $x=0$ in (b) and (c), $\beta=0$ in (a) and (b), $\alpha=0$ in (a) and (c).

23 Non-singular and singular assembly mode changing trajectory for $\beta=0$ (a) and $\alpha=0$ (b) between $T_{1}=$ $[-100,0]$ and $T_{2}=[100,0] \ldots \ldots \ldots \ldots \ldots \ldots \ldots$

2

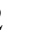

3

\section{List of Tables}

1 Five operation modes of the multi-mode parallel robot. . . . . . . . . . . . . . . . 